\title{
Decoherence can be useful in quantum walks
}

\author{
Viv Kendon* and Ben Tregenna \\ Optics Section, Blackett Laboratory, Imperial College, London, SW7 2BW, United Kingdom
}

(Received 11 September 2002; published 22 April 2003)

\begin{abstract}
We present a study of the effects of decoherence in the operation of a discrete quantum walk on a line, cycle, and hypercube. We find high sensitivity to decoherence, increasing with the number of steps in the walk, as the particle is becoming more delocalized with each step. However, the effect of a small amount of decoherence is to enhance the properties of the quantum walk that are desirable for the development of quantum algorithms. Specifically, we observe a highly uniform distribution on the line, a very fast mixing time on the cycle, and more reliable hitting times across the hypercube.
\end{abstract}

DOI: 10.1103/PhysRevA.67.042315

PACS number(s): 03.67.Lx, 02.50.Ng, 05.40.Fb, 85.35.Be

\section{OVERVIEW}

There is currently great interest within the quantum information community in quantum versions of random walks, because of the possibility that they may produce new, powerful types of quantum algorithms. Apart from Grover's search [1] (quadratic speed up), all the quantum algorithms known until very recently are essentially based on application of the quantum Fourier transform, including Shor's factoring [2], which provides an exponential speed up over the best known classical algorithms. Other types of quantum computation, such as quantum adiabatic computation [3], have not yet been shown to provide exponential speed up over classical methods. Some of the most powerful known classical algorithms are based on classical random walks, so it is a natural question to ask whether there are quantum counterparts that can do even better. For example, a random walk on a general graph can be used to address hard problems such as approximating the permanent, $k$-SAT, and graph connectivity [4-6].

Before attempting to create quantum algorithms from quantum walks, it is first useful to study their properties and dynamics on simpler structures. Several quantum analogs of a classical random walk on discrete lattices or graphs have been proposed. These include discrete time walks both with and without a quantum coin $[7,8]$, and a continuous time walk [9]. The relationship between the continuous time quantum walk and discrete time quantum walks is not fully understood. For the cases where they have been studied on the same graph, they give essentially the same results, e.g., Ref. [10]. Quantum walks on the infinite line, the cycle, and the hypercube have all been solved analytically [10-12], and some bounds are known for more general graphs [11]. On the line and cycle, for most quantities of interest, such as the standard deviation and the mixing time, there is a quadratic speed up over the classical walk. Kempe [13] recently proved that the hitting time to the opposite corner of a hypercube shows an exponential speed up (a possibility also found numerically by Yamasaki et al. [14]). Making an algorithm out of a quantum walk requires significant further work. Two have been proposed very recently, Childs et al.

\footnotetext{
*Electronic address: Viv.Kendon@ic.ac.uk
}

[15] use a continuous time quantum walk to traverse a special graph exponentially faster than any classical algorithm, and Shenvi et al. [16] prove that a coined quantum walk can match Grover's algorithm searching an unstructured database.

Quantum walks themselves (both discrete and continuous time) can be implemented efficiently on a quantum computer [17-19], i.e., it is not necessary to provide a physical implementation of a quantum walk to base an algorithm on them. Nonetheless, quantum walks are interesting in their own right as physical systems in which a precise level of coherent control can be demonstrated. Several direct implementations of discrete walks have been proposed, all with a quantum coin: a walk in the vibration modes of a trapped ion [20], in the phase of the field in a cavity containing an atom [21], and with an atom hopping between traps in an optical lattice [22]. Other than for these physical implementations, and recent work on coin decoherence by Brun et al. [23-25], the effect of decoherence in quantum walks has not previously been studied in any detail.

The key observation of this paper is that, in small doses, rather than degrading the quantum features, decoherence in a coined quantum walk can enhance the desirable quantum speed up, even though overall, quantum walks (consisting as they do of extremely delocalized quantum particles) are highly sensitive to the effects of decoherence. This is very encouraging for the prospects of using quantum walks as the basis of powerful quantum algorithms.

The paper is organized as follows. First we review one of the simplest examples of a quantum walk, the coined walk on a discrete line, and describe the properties of the perfect quantum walk. Then we present our results showing the effects of decoherence in the quantum walk on a line, on a cycle, on a hypercube, and on the "glued trees" graph of Ref. [15].

\section{COINED QUANTUM WALK}

We consider only coined quantum walks on discrete lattices in this paper. Since the classical random walk requires a source of randomness (coin toss) in the dynamics, introducing a quantum coin is a natural way to proceed. For the walk on an infinite line, the total Hilbert space is $H=\mathcal{C}^{2} \otimes \mathcal{H}^{\infty}$, where $\mathcal{H}^{\infty}$ has support on $x \in \mathbb{Z}$. We label the coin states by 
$\{|-1\rangle,|+1\rangle\}$ for "move left" and "move right," respectively, and those of the particle by $|x\rangle$ for position on the line. We write the tensor product states as $|a, x\rangle$, where $a$ $\in\{ \pm 1\}$ is the state of the coin. The unitary operator describing a single step of the walk is

$$
U=S(H \otimes 1),
$$

where $S$ is the conditional shift operator $S|a, x\rangle=|a, x+a\rangle$, and $H$ is the Hadamard operator

$$
(H \otimes 1)|a, x\rangle=(a|a, x\rangle+|-a, x\rangle) / \sqrt{2},
$$

acting on the coin states only, it plays the role of a "coin toss." It can be shown for unbiased quantum walks on a line in which the "coin toss" prepares an equal superposition of states, all such operators are essentially equivalent to the Hadamard operator $[12,26]$. In higher dimensions, when the particle has more than two choices of direction at each step, the choice of unbiased coin-toss operator becomes correspondingly richer $[27,28]$. Unlike the classical case, where each coin toss is independent of the previous coin tosses, in the quantum case, unitarity of the evolution and hence reversibility implies the initial state of the coin has observable consequences at all later times. Specifically, in the following (unless stated otherwise) we will choose the initial state to be $(|+1\rangle+i|-1\rangle) / \sqrt{2}$ which results in a symmetric probability distribution $[12,26,28]$. The dynamics of the quantum walk thus consist of repeated application of the operator $U$ to the particle and coin, resulting in a spreading out on the line, with interference causing the quantum speed up.

The quantum walk on a line has been solved exactly [12] using both real space (path counting) and Fourier space methods. The solutions are complicated, mainly due to the "parity" property, i.e., the solutions must have support only on even-(odd-) numbered lattice sites at even (odd) times. The shape of the probability distribution for the particle position consists of a nearly flat region around the center with the same width as the classical binomial distribution, and oscillating peaks out towards $x= \pm T / \sqrt{2}$. Both quantum and classical distributions are shown in Fig. 1, calculated numerically for $T=200$. The moments can be calculated, for a walk starting at the origin, $\langle|x|\rangle=T / 2$ and $\left\langle x^{2}\right\rangle=(1-1 / \sqrt{2}) T^{2}$ $=\sigma^{2}(T)$. The standard deviation (from the origin) $\sigma(T)$ is thus linear in $T$, in contrast to $\sqrt{T}$ for the classical walk.

\section{DECOHERENCE IN A QUANTUM WALK ON A LINE}

In order to model decoherence in this system, we write the coin-particle dynamics in terms of a density matrix $\rho(t)$ that evolves according to

$$
\rho(t+1)=(1-p) U \rho(t) U^{\dagger}+p \sum_{i} \mathbb{P}_{i} U \rho(t) U^{\dagger} \mathbb{P}_{i}^{\dagger}
$$

Here $P_{i}$ is a projection that represents the action of the decoherence and $p$ is the probability of a decoherence event happening per time step. We took Eq. (3) and evolved it numerically for various choices of $\mathbb{P}_{i}$. Motivated by the likely form of experimental errors, we also modeled an im-

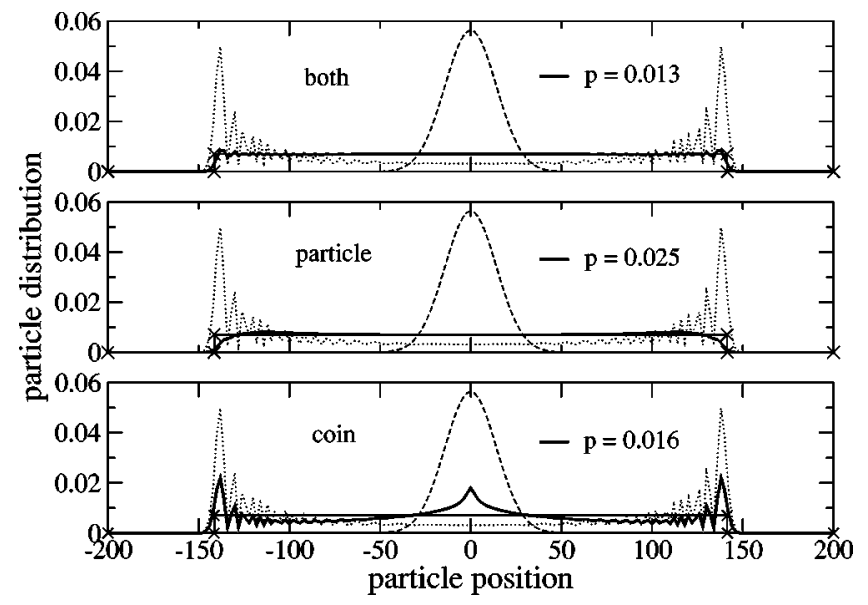

FIG. 1. Distribution of the particle position for a quantum walk on a line after $T=200$ time steps. Pure quantum (dotted), fully classical (dashed), and decoherence at the rate shown on the part of the system indicated by the key (solid). Uniform distribution between $-T / \sqrt{2} \leqslant x \leqslant T / \sqrt{2}$ (crosses) is also shown.

perfect Hadamard operator by applying a Gaussian spread of standard deviation $\sqrt{p} \pi / 4$ about the perfect value of $\pi / 2$ implicit Eq. (2), compare Ref. [27]. An imperfect shift on the particle has been studied in Ref. [22]. In each case we find the same general form for the decay of $\sigma_{p}(T)$ from the quantum to the classical value, with small differences in the rates, as shown in Fig. 2. The slope of $\sigma_{p}(T)$ is finite as $p \rightarrow 0$ and zero at $p=1$. We calculated $\sigma_{p}(T)$ analytically (details in Ref. [29]) for $p T \ll 1$ and $T \gg 1$ for the case where $\mathbb{P}_{i}$ is the projector onto the preferred basis $\{|a, x\rangle\}$ (decohering both particle and coin),

$$
\sigma_{p}(T) \simeq \sigma(T)\left\{1-\frac{p T}{6 \sqrt{2}}+O(p)\right\}
$$

This compares well with simulation data, once a secondorder correction for $\sigma(T)=(1-1 / \sqrt{2})^{1 / 2}(T-1 / T)$ is taken into account. The first-order dependence is thus proportional

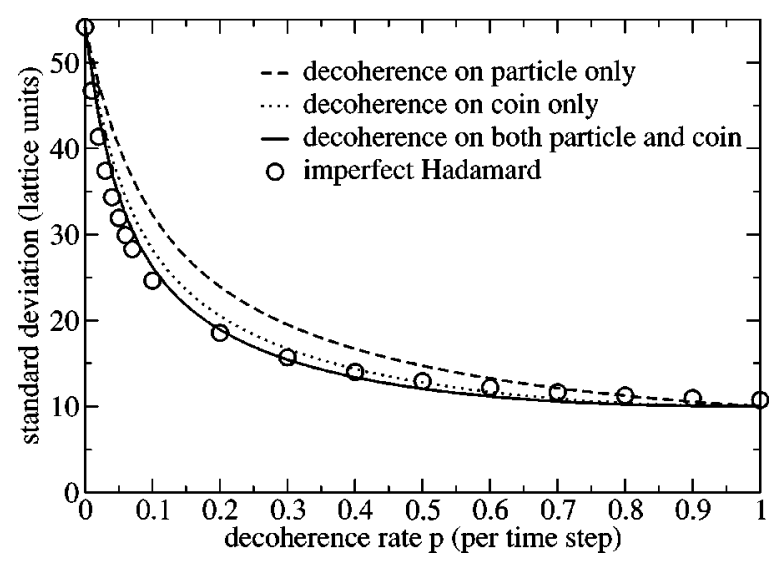

FIG. 2. Standard deviation $\sigma_{p}(T)$ of the particle on a line for different models of decoherence, for $T=100$ time steps. 


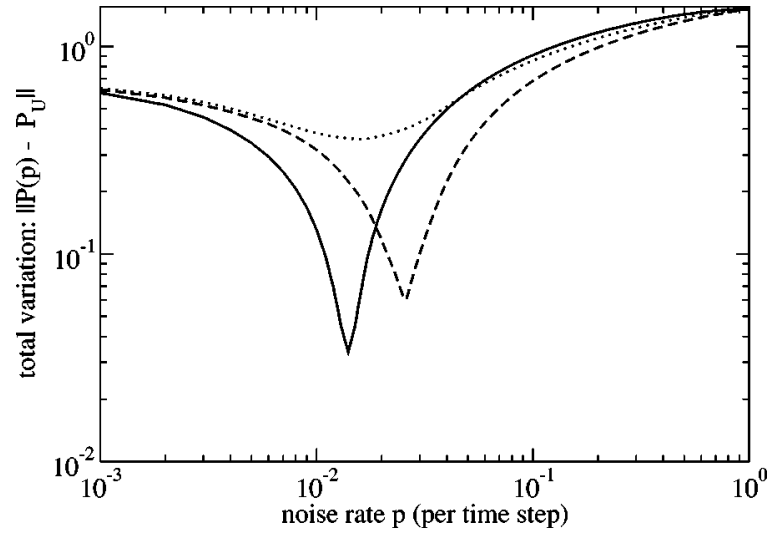

FIG. 3. The total variational distance of the particle position distribution from the uniform distribution for $T=200$ and decoherence on coin (dotted), particle (dashed), or both (solid).

to $p T$, so the sensitivity to decoherence grows linearly in $T$ for a given decoherence rate $p$.

The quantum walk on infinite regular lattices of higher dimension shows a similar decoherence profile, as might be expected, since the standard deviation of a classical random walk is $\sqrt{T}$, independent of dimension.

\section{DISTRIBUTION SHAPE ON A LINE}

The transition from quantum to classical in the standard deviation of the particle position shown in Fig. 2 is qualitatively the same for all types of decoherence examined. However, there are interesting differences in the shape of the distribution of the particle position. The decoherence rate that gives the closest to uniform distribution has been selected and plotted in Fig. 1, along with the pure quantum and classical distributions for comparison. When the particle position is subject to decoherence that tends to localize the particle in the standard basis, this produces a highly uniform distribution between $\pm T / \sqrt{2}$ for a particular choice of $p$. The optimal decoherence rate $p_{u}$ can be obtained by calculating the total variational distance between the actual and uniform distributions,

$$
\nu(p, T) \equiv\left\|P(x, p, T)-P_{u}(T)\right\|_{\mathrm{tv}} \equiv \sum_{x}\left|P(x, p, T)-P_{u}(T)\right|,
$$

where $P(x, p, T)$ is the probability of finding the particle at position $x$ after $T$ time steps, regardless of coin state, and $P_{u}(T)=\sqrt{2} / T$ for $-T / \sqrt{2} \leqslant x \leqslant T / \sqrt{2}$ and zero otherwise. Figure 3 shows $\nu(p, T)$ for $T=200$ with decoherence applied to the coin, particle, and both at once. Decoherence on both particle and coin produces the best uniform distribution, but at a cost of a lower decoherence rate, and sharper minimum, i.e., greater sensitivity to the value of $p$. Walks with decoherence only on the particle can tolerate more variation in the exact decoherence rate, while not achieving such a good uniform distribution. The optimum decoherence rate depends on the number of steps in the walk, we determined numerically that $p_{u} T \simeq 2.6$ for decoherence on both and $p_{u} T \simeq 5$ for decoherence on the particle only. These differences in the quality of the uniform distribution are independent of $p$ and $T$, and provide an order of magnitude (0.6 down to 0.06) improvement in $\nu$ over the pure quantum value. Decoherence just on the coin does not enhance the uniformity of the distribution, as Fig. 1 shows, there is a cusp at $x=0$. However, for finite $T$, there is still a useful window within which even coin only decoherence does not significantly degrade the linear spreading of the walk.

\section{DECOHERENCE ON A CYCLE}

If, instead of an infinite line, the quantum particle is allowed to walk on a cycle of size $N$ points, the appropriate quantity to measure the progress of the walk is the mixing time. We immediately have to modify the classical definition, because, unlike the classical random walk, which mixes to a uniform distribution in the the long time limit, a unitary process such as that evolving the quantum walk does not mix to any limit at large times. We can instead define a timeaveraged particle distribution

$$
\overline{P(x, p, T)}=\frac{1}{T} \sum_{t=0}^{T-1} P(x, p, t)
$$

which does always mix for large enough times $T$ [11]. It is easy to sample from the distribution $\overline{P(x, p, T)}$ : run the walk for some randomly chosen number of steps $0<t<T$ and measure the particle position at that time $t$. The mixing time is then defined as

$$
M_{\epsilon}=\min \left\{T \mid \forall t>T:\left\|\overline{P(x, p, t)}-P_{u}\right\|_{\mathrm{tv}}<\epsilon\right\},
$$

where $P_{u}$ is the limiting (uniform) distribution over the cycle. The mixing time quantifies how long it takes for the time-averaged probability distribution of the particle position to reach its limiting value within a margin of small parameter $\epsilon>0$.

The walk on a cycle is the simplest example of a walk on the Cayley graph of an Abelian group, and was historically the first to be treated analytically [11]. The dynamics of the walk on the cycle are the same as for the walk on a line, with the particle position taken $\bmod (N)$. Aharonov et al. [11] proved an upper bound for the mixing time of $O(N \log N)$. The limiting distribution thus obtained depends on the choice of coin operator [28], in sharp contrast to the classical walk, which always mixes to a uniform distribution. For the Hadamard coin used here, the odd- $N$ cycle mixes to the uniform distribution, but the even- $N$ cycle does not [28].

We numerically evaluated the mixing times for walks on cycles of sizes up to $N \simeq 80$, both for pure states, and in the presence of the same types of decoherence as described in the preceding section for the walk on a line. For odd- $N$ cycles with no decoherence, we find that $M_{\epsilon} \sim N / \epsilon$ as compared to the upper bound of $M_{\epsilon} \sim N \log N / \epsilon$ in Ref. [11]. While we believe that the linear scaling with $N$ is the correct result, obtaining a tighter bound analytically is a tough task, 


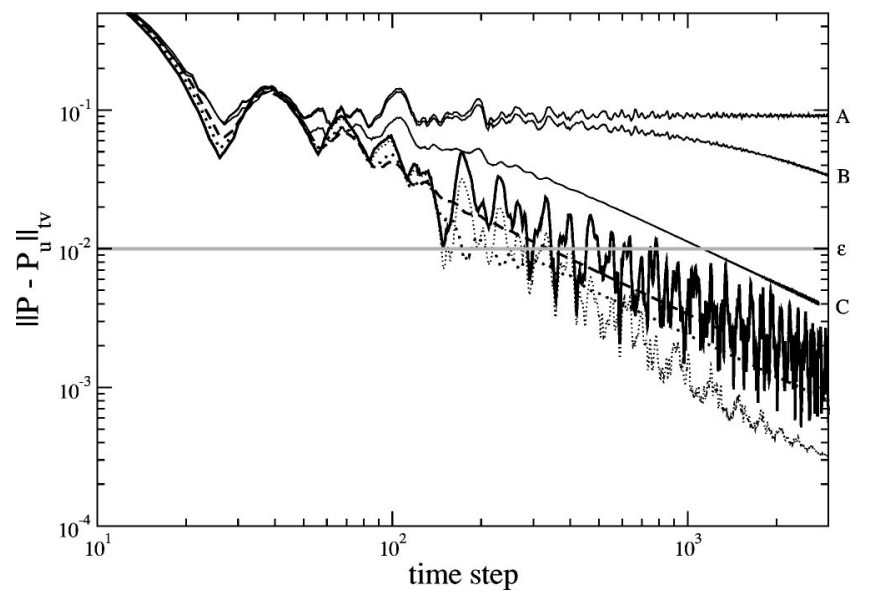

FIG. 4. Difference between the time averaged probability distribution $\overline{P(x, p, T)}$ and the uniform distribution expressed as the total variational distance, Eqs. (5) and (6) for the case with decoherence on both coin and particle. Both axes are logarithmic. The value of $\epsilon$ used in Fig. 5 is shown as a horizontal line. The top three solid lines labeled on right are for $N=22$ with $p=0$ (A), $p=0.001$ (B), $p$ $=0.02(\mathrm{C})$. The lower four lines are for $N=21$ with $p=0$ (solid), $p=0.002$ (dotted), $p=0.01$ (short dashed), $p=0.02$ (long dashed).

because the time averaged probability distribution $\overline{P(x, p, T)}$ is a rather fluctuating quantity, especially for $p=0$, as is illustrated in Fig. 4. In this figure, the quantity $\| \overline{P(x, p, t)}$ $-P_{u} \|_{\text {tv }}$ from Eq. (7) is plotted against time. The time at which the curves last cross the horizontal line at $\epsilon=0.01$ is the mixing time as plotted in Fig. 5. A different choice of $\epsilon$ thus causes a jump in the value of $M_{\epsilon}$ if it happens to touch the next peak in $\left\|\overline{P(x, p, t)}-P_{u}\right\|_{\mathrm{tv}}$.

\section{A. Even- $N$ cycles with decoherence}

Under the action of a small amount of decoherence, the mixing time becomes shorter for all cases, typical results are shown in Fig. 5. Also, decoherence causes the even- $N$ cycle

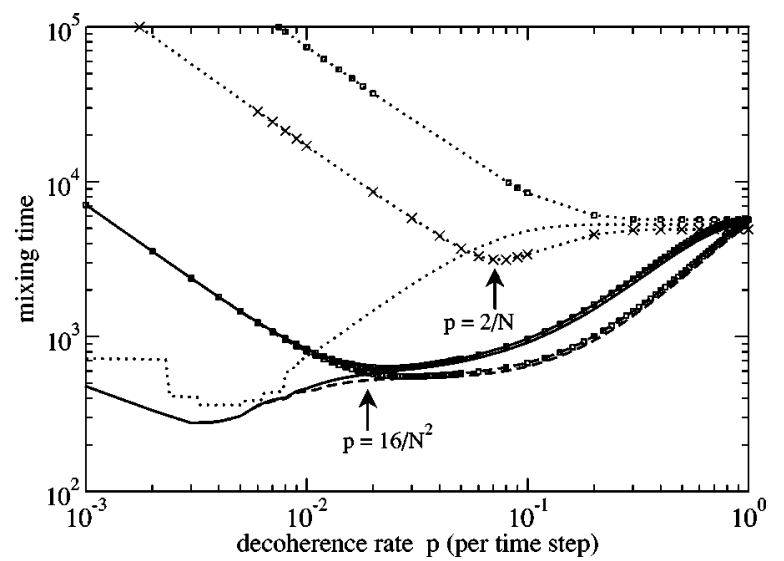

FIG. 5. Numerical data for mixing times on cycles of size $N$ $=29$ and $N=30(\square)$, for coin (dotted), particle (dashed), and both (solid) subject to decoherence, using $\epsilon=0.01$. Also $N=28(\times)$ for coin. Both axes are logarithmic. to mix to the uniform distribution. The asymptotes in Fig. 5 for $N$ even and decoherence on the coin only, for $p<2 / N$, are well fitted by $\epsilon p M_{\epsilon} \simeq N / 4$ for $N$ divisible by 2 , and $\epsilon p M_{\epsilon}$ $\simeq N / 16$ for $N$ divisible by 4 . For larger $p$, the mixing time tends to the classical value of $N^{2} / 16 \epsilon$ [note this is not $\log (1 / \epsilon)$ because we are calculating the average mixing time, Eq. (7)]. Although for $N$ divisible by 4, the (coin-decohered) mixing time shows a minimum below the classical value at $p \simeq 2 / N$, this mixing time is $\gtrsim N^{2} / 32 \epsilon$, i.e., still quadratic in $N$. Thus, although noise on the coin causes the even- $N$ cycle to mix to the uniform distribution, it does not produce a significant speed up over the classical random walk.

For decoherence on the particle position, with $p$ $<16 / N^{2}, \epsilon p M_{\epsilon} \simeq 1 /(N / 2-1)$ for $N$ divisible by 2 , and $\epsilon p M_{\epsilon} \simeq 1 /(N / 4+3)$ for $N$ divisible by 4 . At $p \simeq 16 / N^{2}$, there is a minimum in the mixing time at a value roughly equal to the $(N \pm 1)$-cycle pure quantum mixing time, $M_{\epsilon}^{(\min )}$ $\sim \alpha N / \epsilon$ (with $\alpha$ a constant of order unity). The top three lines in Fig. 5 show how decoherence pulls an even- $N$ cycle down to mix to the uniform distribution at the same rate as the neighboring odd- $N$ cycle. Decoherence on the particle position thus causes the even- $N$ cycle to mix to uniform in linear time for a suitable choice of decoherence rate $p^{(\mathrm{min})}$ $\sim 16 / N^{2}$, independent of $\epsilon$.

\section{B. Odd- $N$ cycles with decoherence}

For all types of decoherence, the odd- $N$ cycle shows a minimum mixing time at a position somewhat earlier than the even- $N$ cycle, roughly $p=2 / N^{2}$, but because of the oscillatory nature of $\overline{P(x, p, T)}$, the exact behavior is not a smooth function of $p$ or $\epsilon$. As decoherence on the particle (or both) increases, the oscillations in $\overline{P(x, p, T)}$ are damped out. The lower set of lines on Fig. 4 shows how both these features affect the mixing time. At $p \simeq 16 / N^{2}$, the mixing time passes smoothly through an inflexion and from then on behaves in a quantitatively similar manner to the adjacent-sized even- $N$ cycles, including scaling as $M_{\epsilon}^{(\min )} \sim \alpha N / \epsilon$ at the inflexion. Thus for $0 \leqslant p \lesssim 16 / N^{2}$ there is a region where the mixing time stays linear in $N$. Our overall conclusion is thus the same as for the walk on a line, there is a useful window within which decoherence enhances rather than degrades the quantum features of the walk.

\section{HYPERCUBE DECOHERENCE}

The hypercube (Boolean $\mathrm{N}$-cube) is also the Cayley graph of a group, and provides a step on the way to quantum walks on more complex structures. Since there are $N$ edges joining at each vertex, we need an $N$-dimensional coin to choose between the possible paths at each step. This opens up a correspondingly larger range of possible unitary operations to use for the coin toss, but a sensible choice is one that respects the symmetry of the underlying graph, and is as far from the identity as possible. For the hypercube, this is the Grover operator, whose elements expressed as an $N \times N$ matrix are defined as $G_{j k}=2 / N-\delta_{j k}$. We also choose a symmetric starting state for the coin with equal weights for all possible directions from the chosen starting node. The high 


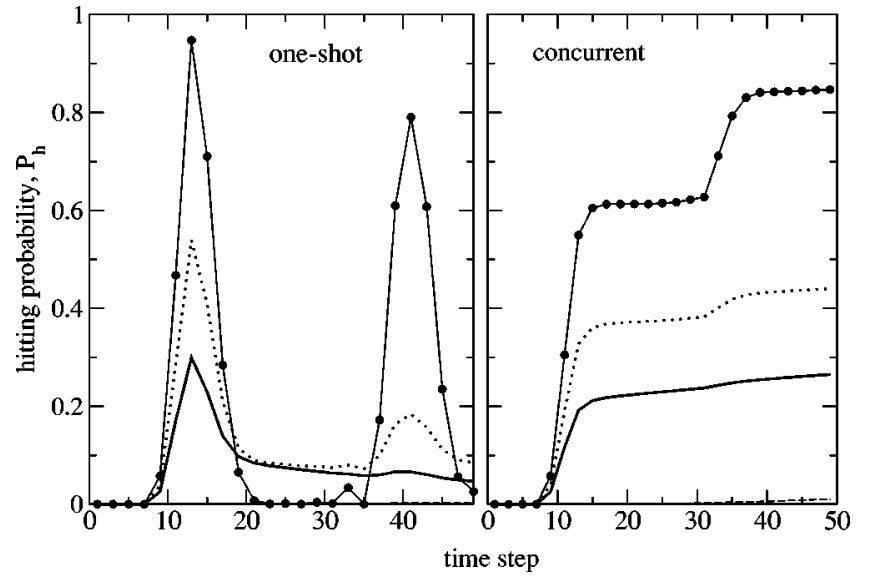

FIG. 6. Hitting probability on a nine-dimensional hypercube for one-shot (left) and concurrent (right), perfect walk (circles), with $p=0.05$ (dotted), $p=0.1 \simeq 1 / 9$ (solid). Classical hitting probability barely visible (dashed).

degree of symmetry in the hypercube allows the quantum walk to be mapped to a walk on a line with a different coin toss operation at each point [10]. While the mixing time for a quantum walk on a hypercube is somewhat worse than a classical random walk, Kempe [13] proved that the hitting time to the opposite corner is polynomial, an exponential speed up over the classical walk.

Kempe discusses two types of hitting times, one shot, where a measurement is made after a predetermined number of steps, and concurrent, where the desired location is monitored continuously to see if the particle has arrived. In each case, the key parameter is the probability $P_{h}$ of finding the particle at the chosen location. We calculated $P_{h}$ numerically and found that all forms of decoherence have a similar effect on $P_{h}$, see Fig. 6, reducing the peaks and smoothing out the troughs. For the one-shot hitting time this is useful, raising $P_{h}$ in the trough to well above the classical value, so it is no longer necessary to know exactly when to measure. For $p$ $\lesssim 1 / N$, the height of the first peak scales as $P_{h}(p)$ $=P_{h}(0) \exp \{-(N+\alpha) p\}$, where $0 \leqq \alpha \lesssim 2$ depending on whether coin, particle, or both are subject to decoherence. An exponential decrease in the presence of decoherence sounds about as bad as it could reasonably be, and for long times, of course, decoherence reduces the walk to classical behavior. However, the hitting times are short, only $\sim N \pi / 2$ steps, and $p \simeq 1 / N$ only lowers $P_{h}$ by a factor of $1 / e$. For algorithmic purposes this is insignificant, only a factor of order unity and thus still exponentially better than classical. (Standard amplification techniques can be used to bring the hitting probability as close to 1 as desired.) Note also, that the size of the graph (measured in number of nodes) is exponential in $N$, so the decoherence has only a linear effect measured in terms of the size of the graph.

Continuous monitoring of the target location as in the concurrent hitting time is already a sort of controlled decoherence, note that the height of the initial peak at $\sim N \pi / 2$ steps is only about $2 / 3$ that of the one-shot hitting probability. No extra features are produced by the addition of unse- lective decoherence, but there is still a range of $0<p \leqq 1 / N$ within which the quantum speed up is preserved. Note that in both the one-shot and concurrent cases, $p \simeq 1 / N$ is a critical damping rate, smoothing out the second peak [shown at around $40(\equiv 3 N \pi / 2)$ steps in Fig. 6].

\section{VII. "GLUED TREES" WALK}

The graph used by Childs et al. [15] is similar to the hypercube in that it can also be mapped to a walk on a line. It consists of two identical binary trees of depth $N$, glued at their branches by a randomly distributed set of edges (two per node, so the degree of the graph is three except for the roots of the trees). They use a continuous time walk for their algorithm, but a discrete quantum walk using a threedimensional Grover coin operator has essentially similar properties $[19,28]$. The effects of decoherence are similar to the hypercube. The peak in the probability of finding the particle at the exit node occurs after $2 N+3$ steps, and is around 0.6 for a pure walk. Decoherence reduces this peak exponentially (in $N$ ) while spreading out the range of time steps over which the probability is significantly (exponentially) larger than the classical value.

\section{CONCLUSIONS}

One of the generic ways in which classical random walks are applied to algorithms is in the guise of Monte Carlo Markov chains and their variants, to sample an exponentially large problem space to estimate statistical properties of the system. Fast mixing times and uniform sampling are necessary properties of the random walk process for it to perform efficiently. This is the sense in which we propose that a small amount of decoherence in a walk on the line or the cycle is beneficial, producing more uniform distributions (line) and faster mixing to a uniform distribution (cycle).

On the hypercube and "glued trees" walks, the key quantum property exploited by the examples in the literature is the opposite of a uniform distribution, the ability of a quantum walk to continue its forward march through the graph despite the many possible "wrong turns" a classical random walk gets lost in. For the examples given, it is easy to determine a priori the best time to check for the particle having found the target node. But for more complex graphs this may not be easy to calculate, so having a wider window of opportunity to successfully detect the particle could be an advantage. Note that the concurrent hitting time is doing exactly this in a more precise way by monitoring the target node at every time step. For these type of walks, we do not claim that decoherence gives a major advantage, only that it is not detrimental for small decoherence rates.

We have also found numerically that the walk on a cycle mixes in linear time [compared to the upper bound of $O(N \log N)$ proved in Ref. [11]], and show why it is hard to prove a tighter bound analytically because the quantities involved are highly fluctuating.

Overall, these results are quite promising for the development of further quantum algorithms, and for the practical implementation of quantum computing. They are also en- 
couraging for the experimental implementation of quantum walks as proposed in Refs. [20-22]. For the modest number of steps in the proposals, our results suggest a very reasonable range in which quantum effects can be observed experimentally. Finally, it is also an intriguingly counterintuitive result in its own right, worthy of further study in the context of the transition from quantum to classical.

\section{ACKNOWLEDGMENTS}

We thank Peter Knight, Richard Maile, Will Flanagan, Julia Kempe, Todd Brun, Hilary Carteret, John Watrous, and Dorit Aharonov for useful discussions. This work was funded by the UK Engineering and Physical Sciences Research Council.
[1] L.K. Grover, in Proceedings of the 28th Annual Symposium on the Theory of Computing (ACM Press, New York, 1996), pp. 212-219.

[2] P.W. Shor, SIAM (Soc. Ind. Appl. Math.) J. Sci. Stat. Comput. 26, 1484 (1997),

[3] E. Farhi, J. Goldstone, S. Gutmann, and M. Sipser, e-print quant-ph/0001106.

[4] M. Jerrum, A. Sinclair, and E. Vigoda, in Proceedings of the 33rd Symposium on the Theory of Computing (ACM Press, New York, 2001), pp. 712-721.

[5] U. Schöning, in Proceedings of the 40th Annual Symposium on Foundations of Computer Science (IEEE Computer Society Press, Los Alamitos, CA, 1999), pp. 17-19.

[6] R. Motwani and P. Raghavan, Randomized Algorithms (Cambridge University Press, Cambridge, UK, 1995).

[7] Y. Aharonov, L. Davidovich, and N. Zagury, Phys. Rev. A 48, 1687 (1992).

[8] J. Watrous, J. Comput. Syst. Sci. 62, 376 (2001),

[9] E. Farhi and S. Gutmann, Phys. Rev. A 58, 915 (1998),

[10] C. Moore and A. Russell, in Proceedings of the 6th International Workshop on Randomization and Approximation Techniques in Computer Science (RANDOM '02), edited by J.D.P. Rolim and S. Vadhan (Springer, New York, 2002), pp. 164178.

[11] D. Aharanov, A. Ambainis, J. Kempe, and U. Vazirani, in Proceedings of the 33rd Symposium on the Theory of Computing (Ref. [4]), pp. 50-59.

[12] A. Ambainis, E. Bach, A. Nayak, A. Vishwanath, and J. Watrous, in Proceedings of the 33rd Symposium on the Theory of Computing (Ref. [4]), pp. 60-69.

[13] J. Kempe, e-print quant-ph/0205083.

[14] T. Yamasaki, H. Kobayashi, and H. Imai, in Proceedings of the
Third International Conference on Unconventional Models of Computation, 2002, Kobe, Japan, edited by C. Calude, M.J. Dinneen, and F. Peper (Springer, New York, 2002), pp. 315330.

[15] A.M. Childs, R. Cleve, E. Deotto, E. Farhi, S. Gutmann, and D.A. Spielman, e-print quant-ph/0209131.

[16] N. Shenvi, J. Kempe, and K.B. Whaley, Phys. Rev. A (to be published), e-print quant-ph/0210064.

[17] J. Kempe (private communication).

[18] D. Aharonov and A. Ta-Shma, e-print quant-ph/0301023.

[19] J. Watrous (private communication).

[20] B.C. Travaglione and G.J. Milburn, Phys. Rev. A 65, 032310 (2002).

[21] B. Sanders, S. Bartlett, B. Tregenna, and P.L. Knight, Phys. Rev. A 67, 042305 (2003).

[22] W. Dür, R. Raussendorf, H.-J. Briegel, and V.M. Kendon, Phys. Rev. A 66, 052319 (2002),

[23] T.A. Brun, H.A. Carteret, and A. Ambainis, Phys. Rev. Lett. (to be published), e-print quant-ph/0208195.

[24] T.A. Brun, H.A. Carteret, and A. Ambainis, Phys. Rev. A (to be published), e-print quant-ph/0210161.

[25] T.A. Brun, H.A. Carteret, and A. Ambainis, Phys. Rev. A 67, 032304 (2003).

[26] E. Bach, S. Coppersmith, M.P. Goldschen, R. Joynt, and J. Watrous, e-print quant-ph/0207008.

[27] T.D. Mackay, S.D. Bartlett, L.T. Stephenson, and B.C. Sanders, J. Phys. A 35, 2745 (2002).

[28] B. Tregenna, W. Flanagan, R. Maile, and V. Kendon (unpublished).

[29] B. Tregenna and V. Kendon, in Quantum Communication, Measurement \& Computing (QCMC'02) (Rinton Press, Princeton, NJ, 2002). 